\title{
Optic Glomeruli and Their Inputs in Drosophila Share an Organizational Ground Pattern with the Antennal Lobes
}

\author{
Laiyong Mu, ${ }^{1}$ Kei Ito, ${ }^{2}$ Jonathan P. Bacon, ${ }^{3}$ and Nicholas J. Strausfeld ${ }^{1}$ \\ ${ }^{1}$ Department of Neuroscience, University of Arizona, Tucson, Arizona 85721, ${ }^{2}$ Center for Bioinformatics, Institute of Molecular and Cellular Biosciences, \\ University of Tokyo, Bunkyo-Ku, Tokyo 113-0032, Japan, and ${ }^{3}$ School of Life Sciences, University of Sussex, Falmer, Brighton BN1 9QG, East Sussex, United \\ Kingdom
}

Studying the insect visual system provides important data on the basic neural mechanisms underlying visual processing. As in vertebrates, the first step in visual processing in insects is through a series of retinotopic neurons. Recent studies on flies have found that these converge onto assemblies of columnar neurons in the lobula, the axons of which segregate to project to discrete optic glomeruli in the lateral protocerebrum. This arrangement is much like the fly's olfactory system, in which afferents target uniquely identifiable olfactory glomeruli. Here, whole-cell patch recordings show that even though visual primitives are unreliably encoded by single lobula output neurons because of high synaptic noise, they are reliably encoded by the ensemble of outputs. At a glomerulus, local interneurons reliably code visual primitives, as do projection neurons conveying information centrally from the glomerulus. These observations demonstrate that in Drosophila, as in other dipterans, optic glomeruli are involved in further reconstructing the fly's visual world. Optic glomeruli and antennal lobe glomeruli share the same ancestral anatomical and functional ground pattern, enabling reliable responses to be extracted from converging sensory inputs.

\section{Introduction}

Visual processing allows animals to negotiate their environment and direct their behaviors. The optic lobes of Drosophila reconstruct salient features of the taxon's visual ecology by processing optic flow and distinguishing static features. Although Drosophila has a less elaborate nervous system than most vertebrates, many features are shared (Sanes and Zipursky, 2010). Visual processing in Drosophila involves sequential interactions by stratified networks in the medulla, with retinotopic neurons that supply information to two deeper retinotopic neuropils, the lobula and the lobula plate. The latter is a tectum-like neuropil in which large field tangential cells integrate signals from achromatic relays and respond to the orientation and direction of optic flow (Schnell et al., 2010). In contrast, the lobula is a cortex-like neuropil (Ramon y Cajal and Sánchez, 1915) that comprises many palisades of lobula columnar neurons (LCNs). These are comparable to pyramidal cells of the mammalian striate cortex (Strausfeld, 1970). The next level of the fly's visual system is glomerular. Axons from each palisade of LCNs group into a unique bundle that targets a unique glomerulus in the brain's lateral protocerebrum (Strausfeld and Bacon, 1983; Strausfeld and Lee, 1991; Otsuna and Ito, 2006; Strausfeld and Okamura, 2007). This deepest part of the visual system shares a neural organization with the glomerular

\footnotetext{
Received January 16, 2012; revised March 1, 2012; accepted March 7, 2012.

Author contributions: L.M., J.P.B., and N.J.S. designed research; L.M. performed research; L.M. and K.I. contributed unpublished reagents/analytic tools; L.M. analyzed data; L.M., J.P.B., and N.J.S. wrote the paper.

This work was supported by National Institutes of Health National Center for Research Resources Grant R01 08688 and Air Force Office of Scientific Research Grant 300900. We thank Dr. Rachael Wilson for suggestions on the whole-cell patch recording setup, and Dr. Jie Pu for advice on data analysis methods.

Correspondence should be addressed to Laiyong Mu, Department of Neuroscience, 1040 East 4th St, University of Arizona, Tucson, AZ 85721. E-mail: mul@email.arizona.edu.

DOI:10.1523/JNEUROSCI.0221-12.2012

Copyright $\odot 2012$ the authors $\quad 0270-6474 / 12 / 326061-11 \$ 15.00 / 0$
}

antennal lobes, composing a network of local interneurons (LINs) and projection neurons (Strausfeld and Bacon, 1983; Strausfeld et al., 2007).

Such commonality raises fundamental questions about principles of sensory-system organization. Optic glomeruli, and the organization of local interneurons within them, are the protocerebral (segmental) homologs of the deutocerebral antennal lobe (Strausfeld et al., 2007), in which each glomerulus receives converging inputs from olfactory sensory neurons expressing the same odorant-receptor gene (Gao et al., 2000; Vosshall et al., 2000). Noisy olfactory signals from receptors are refined through interactions among glomeruli through local interneurons and are then relayed to higher centers by projection neurons (Laurent, 2002; Wilson, 2008). Whereas olfactory receptor neurons encode only one modality, the identities of specific molecular ligands, compound-eye photoreceptors, encode many submodalities, such as intensity changes, e-vectors, and spectral properties. Features of the visual world, comparable in their specificity to the encoding of specific odorants, are not detected at the receptor level but are reconstructed by subsequent retinotopic layers relaying to palisades of LCNs. There are as many unique palisades of these neurons as there are optic glomeruli, and organizational correspondence of optic glomeruli and antennal lobe glomeruli suggest comparable network organization in which each glomerulus is supplied by a characteristically defined input relaying a specific sensory feature of the visual or olfactory environment.

To test whether optic glomeruli are indeed functionally comparable to antennal-lobe glomeruli, we focused on LCNs consisting of clones of $\sim 40$ identical neurons and their postsynaptic targets. Responses of single lobula output neurons, LINs, and projection neurons were obtained using whole-cell patch-clamp recording methods developed for the olfactory system (Wilson 
and Laurent, 2005). Our results provide the first electrophysiological evidence for convergent processing in an optic glomerulus. Convergent signals at a glomerulus are disambiguated at, and enhanced by, the follower relay neuron. Our data also show the participation in this convergence by local interneurons associated with that specific glomerulus.

\section{Materials and Methods}

Flies. Flies were raised on standard cornmealagar medium. The experimental flies were 2- to 7-d-old adult female Drosophila melanogaster of the UAS-mCD8::GFP A307 line or the progeny of crossing GAL4 enhancer-trap lines NP3045 (Otsuna and Ito, 2006) and NP5092 with UAS-GFP reporter line UAS-GFP S65T. Single-neuron somata of lobula complex output clones labeled in NP3045 and NP5092 were targeted for patch-clamp recording (Fig. 1). Recordings were also obtained from a LIN of the glomerulus receiving inputs from one clone in NP5092 and from the major projection neuron [the giant fiber (GF)] associated with this glomerulus, resolved in the GFP line UAS-mCD8::GFP A307.

Animal preparation. Our animal setup (Fig. 2 ) is adapted from that reported by Wilson and Laurent (2005). Flies were inserted into a hole located in the center of a square of aluminum foil, which was attached to the center of a Petri dish. The Petri dish was then fastened to a fixed stage underneath an Olympus BX51WI microscope. A small amount of glue (Super Glue) and wax was used to suspend the fly in the hole. The dorsal-ventral axis of the animal's body was perpendicular to the horizontal plane defined by the foil. The position of the fly's head was adjusted to a standard position $( \pm<2$ $\mathrm{mm}$ ) using the coordinate lines on the Petri dish and the vernier of the microscope stage. The head capsule of the fly was fixed with its posterior plane horizontal. Thus, the back of the head could be bathed in saline while the eyes in air received unimpeded visual stimuli. The optic lobe and/or the protocerebrum of the brain was exposed by removing the posterior head cuticle and then bathing in extracellular saline $[103 \mathrm{~mm}$ $\mathrm{NaCl}, 3 \mathrm{~mm} \mathrm{KCl}, 5 \mathrm{~mm} \mathrm{~N}$-tris (hydroxymethyl) methyl-2-aminoethanesulfonic acid, $26 \mathrm{~mm} \mathrm{NaHCO}_{3}, 1 \mathrm{~mm} \mathrm{NaH}_{2} \mathrm{PO}_{4}, 1.5 \mathrm{~mm} \mathrm{CaCl}_{2}, 4 \mathrm{~mm} \mathrm{MgCl}_{2}, 10$ $\mathrm{mm}$ trehalose, $10 \mathrm{~mm}$ glucose, and $2 \mathrm{~mm}$ sucrose, adjusted to $275 \mathrm{mOsm}, \mathrm{pH}$ equilibrated at $\sim 7.3$ when bubbled with $95 \% \mathrm{O}_{2} / 5 \% \mathrm{CO}_{2}$ ] (Wilson and Laurent, 2005). Papain (15 U/ml, Sigma) activated by $1 \mathrm{~mm}$ L-cysteine (Sigma) (Gu and O'Dowd, 2007) was locally applied above the somata of the GFP-labeled LCNs or GFs through a blunt glass electrode for $\sim 1 \mathrm{~min}$. Then the trachea and sheath above the target area were removed with forceps (FST 5SF), or a sharp broken glass electrode, to expose the somata of the labeled neurons. The brain was continuously perfused with the extracellular saline $\left(95 \% \mathrm{O}_{2} / 5 \% \mathrm{CO}_{2}\right.$ bubbled) throughout the recording.

Visual stimuli. Visual stimuli were presented through a customized, flat, light-emitting diode (LED) arena (Reiser and Dickinson, 2008) composed of $8 \times 7$ LED panels. MATLAB 7.9 MathWorks and the controller panel were used to program and execute visual stimuli on the LED arena, which was mounted at $45^{\circ}$ under the immobilized fly. The long axis of the arena was adjusted to be parallel to the long axis of the fly thorax (Fig. $2 A$ ). The LED arena provided one eye with a $67^{\circ}$ (vertical) by $59^{\circ}$ (horizontal) visual field (Fig. 2 B). Stimuli included full-field flicker, square-wave gratings (spatial frequency of $8.4 \%$ cycle, velocity of $29.4 \%$ s), sinusoidal gratings (spatial frequency of $33.5^{\circ}$ cycle, velocity of $39^{\circ}$ or $29.4^{\circ} / \mathrm{s}$ ), moving bars (a black bar on a white background or a white bar on a black background, width of $16.7^{\circ}$, velocity of $39^{\circ} / \mathrm{s}$ or $29.4^{\circ} / \mathrm{s}$ ), and static patterns of square-wave gratings with different orientations (Fig. 2C). The Michelson contrast value of the patterns was 1 . Sequences of mixed stimuli patterns were used as follows: (1) $0.5 \mathrm{~Hz}$ flicker (5 s duration); (2) square-wave grating motion in eight different directions; (3) a single bar moving in four different directions; (4) sinusoidal grating motion in four different directions (stimuli 2, 3, and 4 were stationary for 1 or $2 \mathrm{~s}$ and then moved for 2 or $5 \mathrm{~s}$ ); and (5) an expanding (looming)/retracting square black block at the center of the LED panels $(40 \%)$. When the recording was stable, stimuli were repeated an additional one to four times, either immediately before moving to the next stimulus pattern or after cycling through the entire stimulus set. The direction and orientation of stimuli described in Results refer to the head in its normal position.

Whole-cell patch-clamp recording. The somata of GFP-labeled target neurons were patched under visual control through an Olympus BX51WI microscope with infrared-differential interference contrast optics. We also made whole-cell patch-clamp recordings of some non-GFP neurons in the brain by targeting the somata with interference optics alone. The solution within the patch-clamp electrode $(10-13 \mathrm{M} \Omega)$ consisted of $140 \mathrm{~mm}$ potassium aspartate, $1 \mathrm{~mm} \mathrm{KCl}, 4 \mathrm{~mm}$ MgATP, $0.5 \mathrm{~mm}$ $\mathrm{Na}_{3}$ GTP, 10 mм HEPES, and 1 mм EGTA, pH 7.3 (265 mOsm) (Wilson and Laurent, 2005). Biocytin (0.5\%) was added for subsequent identifi- 

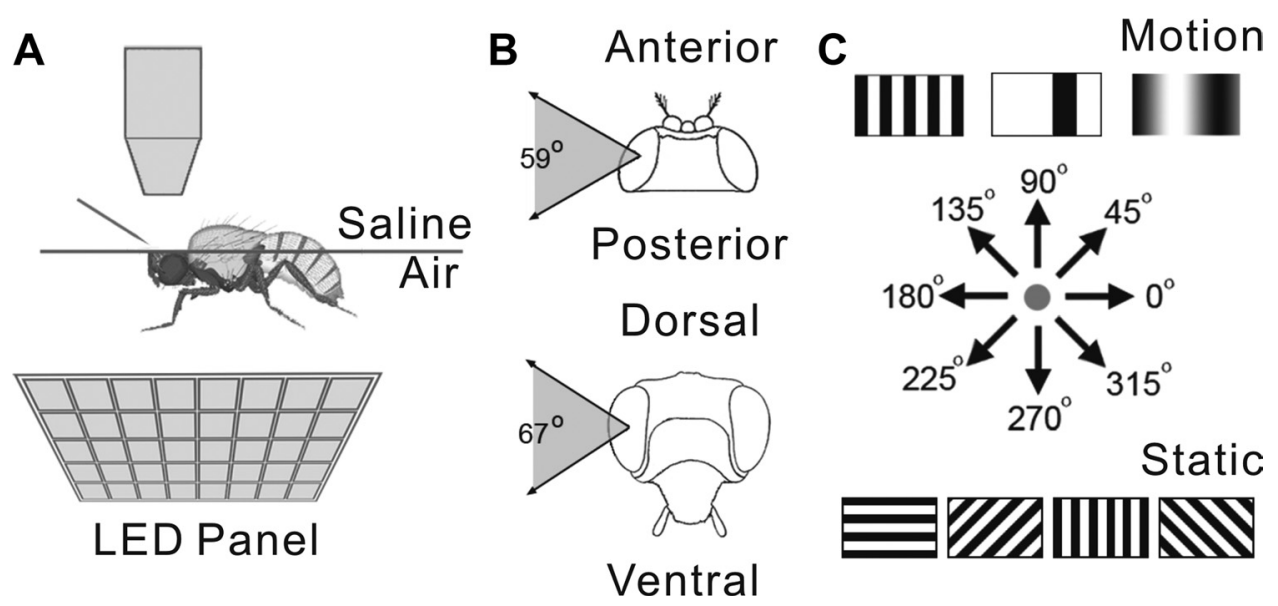

Figure 2. Experimental setup. $A$, The whole-cell patch-recording setup. Flies were inserted into a square of aluminum foil attached to a Petri dish. The dorsal-ventral axis of the animal's body was fixed perpendicularly to the horizontal plane defined by the foil. The head of the fly was bent downward until the posterior plane of its head was horizontal. The back of the head was bathed in saline, and the eyes remained in air to receive visual stimuli from LED panel beneath. $\boldsymbol{B}$, The subtended visual field was $59^{\circ}$ horizontally and $67^{\circ}$ vertically. $\boldsymbol{C}$, Visual stimuli included flicker, three types of motion pattern, square grating, single bar, and sinusoidal grating, moving in four or eight different directions, and static square gratings at four different orientations.

cation of recorded cells. Voltage was recorded with Spike2 6.0 software (Cambridge Electronic Design) in current-clamp mode through an Axopatch 200B amplifier (Molecular Devices), low-pass filtered at $5 \mathrm{kHz}$, and digitized at $10 \mathrm{kHz}$ with a Power 1401 digitizer (Cambridge Electronic Design). To subsequently confirm the identity of a recorded cell, only one cell was patch-clamped in each animal.

Immunohistology and anatomical reconstruction. After recording, the fly's brain was dissected and fixed in $4 \%$ formaldehyde in PBS for $2 \mathrm{~h}$ at room temperature or overnight at $4^{\circ} \mathrm{C}$. After six rinses with PBST $(0.5 \%$ Triton X-1000 in PBS) for 15 min each, the brains were blocked with $10 \%$ goat serum for $2 \mathrm{~h}$ at room temperature, then incubated with 1:1000 rabbit antibody to GFP (Invitrogen) overnight at $4^{\circ} \mathrm{C}$. After six rinses with PBST for $15 \mathrm{~min}$ each, the brains were incubated overnight at $4^{\circ} \mathrm{C}$ with 1:1000 goat antibody to rabbit:Cy5 (Invitrogen) to visualize GFPlabeled neurons or 1:1000 streptavidin:Cy3 (Jackson ImmunoResearch) to visualize the biocytin-filled cells. After six rinses in PBST for $15 \mathrm{~min}$ each, brains were mounted in Vectashield (Vector Laboratories) on a slide. Images of brains were obtained from a Zeiss AxioPlan2 confocal microscope with a $40 \times$ oil-immersion objective. Stacks (1-2 $\mu \mathrm{m}$ slices) of images were used to reconstruct the anatomy of the recorded neurons in Adobe Photoshop CS2.

Data analysis. Because electrophysiological records of the lobula output neurons revealed both fast and slow membrane potential fluctuations other than spikes, we used power spectrum analysis to quantify the activity of the lobula output neurons. Results are reported only for neurons that had input resistance $>5 \mathrm{G} \Omega$, which was our threshold criterion for the goodness of the seal and, therefore, the quality of the electrophysiological recording. Time-frequency analysis was conducted in MATLAB 7.9 (MathWorks) using a program written by author L.M. based on an algorithm published by Cohen et al. (2009). Time-frequency decomposition was computed through wavelet analysis, by which the recording was convolved with a set of complex Morlet wavelets, defined as a Gaussian-windowed complex sine wave as follows: $e^{i 2 \pi t f} e^{-t^{2} /\left(2 \sigma^{2}\right)}$, where $t$ is time and $f$ is frequency, which ranges from 2 to $80 \mathrm{~Hz}$ in 20 logarithmically spaced steps; $\sigma$ defines the width of each frequency band and was set according to $5 /(2 \pi f)$; and 5 is the number of wavelet cycles that provides a balance between time and frequency resolution. After convolving the signal with the wavelets, power was defined as the modulus of the resulting complex signal $z(t)$ (power time series: $p(t)=\operatorname{real}[z(t)]^{2}+$ imag $[z(t)]^{2}$ ). The baseline was defined as the average power in the second before the beginning of each stimulus. The final power time sequences were normalized to a decibel scale, $10^{\star} \log _{10}$ (response/baseline), which allows a direct comparison across frequency bands. Furthermore, the averaged $\mathrm{dB}$ power from 2 to $80 \mathrm{~Hz}$ through a given time period can be calculated from the time-frequency analysis. We also used an alternative method to calculate time-averaged power spectra (see Fig. $6 C$ ), which directly uses the discrete Fourier transform function, $f f t$, in MATLAB 7.9 (MathWorks).

The extracted power data were statistically analyzed to examine whether these neurons show selective responses to particular visual stimuli. To test the effect of flicker, one-way repeated-measures ANOVA was conducted using time (time windows $a \sim e$; see Fig. $7 B, C$ ) as the sole factor. If a significant effect of time was found, multiple comparisons among pairs of time windows (e.g., time windows $a$ vs $b$, time windows $b$ vs $c$ ) were made using the Holm step-down procedure (Holm, 1979) to control the overall type I error level. To test the responses of motion stimuli and static patterns, data were analyzed by two-way repeatedmeasures ANOVA with direction/orientation and time (200 ms before vs $200 \mathrm{~ms}$ after the onset of the stimuli) as factors. If significant directionby-time or orientation-by-time interaction effects were found, it indicated that the neurons did respond to the stimuli differently at the different directions (or orientations). To determine which directions or orientations caused the power of a neuron's membrane potential fluctuations to change significantly following the onset of the stimuli, a test of the simple effect of time was conducted at each direction or orientation. For all analyses, effects were considered significant at $p \leq 0.05$ and marginally significant at $p \leq 0.10$.

\section{Results}

\section{Nerve cell morphologies relate to neural coding}

Neurons in the Drosophila brain produce spiking, nonspiking, and mixed spiking responses to visual stimuli. Figure $3 A$ shows a neuron responding with tonic spiking to flicker, with its firing rate increasing in response to decrements of illumination and decreasing in response to increments. In contrast, the neuron depicted in Figure $3 B$ responds with depolarizing membrane potentials, but not spikes, in response to intensity decrements. Similar distinctions have been routinely documented in the fly Phaenicia, in which the smallest neurons with the thinnest axons conduct information by graded potentials and larger neurons conduct by action potentials, or mixed graded and action potentials (Gilbert and Strausfeld, 1992; Douglass and Strausfeld, 1995, 1996; Okamura and Strausfeld, 2007). An example of a mixed response is shown by the giant vertical motionsensitive (VS) neurons in the lobula plate of Drosophila (Fig. 3C). VS neurons in larger flies display mixed responses similar to those in Drosophila (Douglass and Strausfeld, 1996; Joesch et al., 2008; Maimon et al., 2010)

We recorded and anatomically identified different neurons in the visual system to determine whether these physiological spiking and nonspiking characteristics of individual neurons are cor- 
related with their anatomical features. Output neurons from the lobula conduct only by electrotonic transmission; they show both fast and slow membrane potential fluctuations but never spike, even when injected with depolarizing current (Fig. 4A). These neurons have an axon length of $80-90 \mu \mathrm{m}$, and a diameter of $<0.5 \mu \mathrm{m}$. In contrast, either mixed or exclusively spiking responses are elicited in neurons that occur as bilateral pairs of "unique" cells or as very small populations of two to eight identical neurons. These neurons have axon diameters of at least $0.5 \mu \mathrm{m}$ and axons ranging from 80 to $410 \mu \mathrm{m}$ in length (Fig. $4 B, C$ ). Descending neurons that extend from the brain to the thoracic ganglia, and that also occur as pairs, have 500- to 700- $\mu \mathrm{m}$-long axons, with axon diameters ranging between 1.5 and $3 \mu \mathrm{m}$. Such neurons conduct by action potentials (Fig. 4D).

There is a clear association between anatomical and physiological characteristics of this sample of fly visual interneurons. For example, the short narrow axons of the LCNs are nonspiking. As reported by Faisal and Laughlin (2007), such smalldiameter processes are subject to channel noise, which corrupts spiking transmission. Encoding of visual parameters by such thin axons may be less reliant on the efficiency of signal propagation by single neurons and instead relies on the collaborative encoding by subsets of neurons that converge onto a common postsynaptic target. This is confirmed by findings described below.

Signal reliability and enhancement at the optic glomerulus We focused on two clones of lobula complex columnar neurons: type 1 lobula columnar neurons (L1CNs), labeled in the GAL4 line NP3045 (Otsuna and Ito, 2006), and type 2 lobula plate lobula columnar neurons (LPL2CNs), labeled in the Gal4 line NP5092. These neurons target two distinct optic glomeruli (Fig. $1 A, B)$. Axons of LPL2CNs converge with terminals from a third clone, lobula Col A cells (Strausfeld and Hausen, 1977), at the GF glomerulus, so-called because its cluster of projection neurons includes the GF, first described by Koto et al. (1981). Physiological recordings were obtained from the LPL2CN lobula complex output neurons, the glomerular LIN associated with the GF glomerulus, and the GF itself.

The LPL2CN (Fig. 5Ai) is one of an isomorphic population, which has been identified in several dipterous species (Strausfeld and Gilbert, 1992). In Drosophila, the LPL2CN clone comprises 40 identical sibling neurons spaced one to every three retinotopic columns. Typical of such ensembles, each neuron has a conical dendritic field extending through the depth of the lobula plate and is linked by a stout process to a narrow but deep dendritic field in the lobula. The dendritic processes of the LPL2CN subtend an oval configuration of six retinotopic columns from the medulla, with each column representing a set of optically coherent R1-R6 photoreceptors, each of which has an acceptance angle of $\sim 5^{\circ}-6^{\circ}$ (Heisenberg and Wolf, 1984). Each LPL2CN thus sub- tends a circular area of the visual panorama $\sim 30^{\circ}$ wide. Together, the $40 \mathrm{LPL} 2 \mathrm{CNs}$, the neighboring cells of which have overlapping visual fields, subtend the entire retina of one eye.

Typical of lobula complex output neurons, responses of the LPL2CN are subtle and, without power-spectrum analysis (see Materials and Methods), are not clearly resolved from membrane-potential fluctuations. This typical aspect of LCNs is considered in greater detail later. Power spectrum analysis of the LPL2CN (Fig. 5Aii) shows that the neuron usually, but not invariably, responds to a looming stimulus expanding over the retina. In contrast, the LIN of the glomerulus in which LPL2CNs terminate (Fig. $5 \mathrm{Bi}$ ) shows an unambiguous and rapidly adapting response to the looming stimulus (Fig. 5Bii). Furthermore, the response of the LIN to slow full-field flicker shows that intensity decrements, such as are incurred by a looming dark stimulus, initiate a larger depolarization than do intensity increments (Fig. 5 Biii). The same looming stimulus also elicits corresponding depolarization in one of the major projection neurons of this glomerulus, the GF (Fig. 5C). These findings suggest that although any single lobula complex output neuron LPL2CN unreliably encodes the looming stimulus, encoding by the LIN involves signal averaging, and thus noise reduction, and the encoded signal relayed to the GF. In larger species of Diptera, and likely in Drosophila, these are integrated with signals representing other sensory modalities (Bacon and Strausfeld, 1986). We propose that encoding of a visual primitive (Marr, 1976) by an ensemble of lobula complex outputs results in the amplified LIN response, which is relayed to the projection neurons of the 

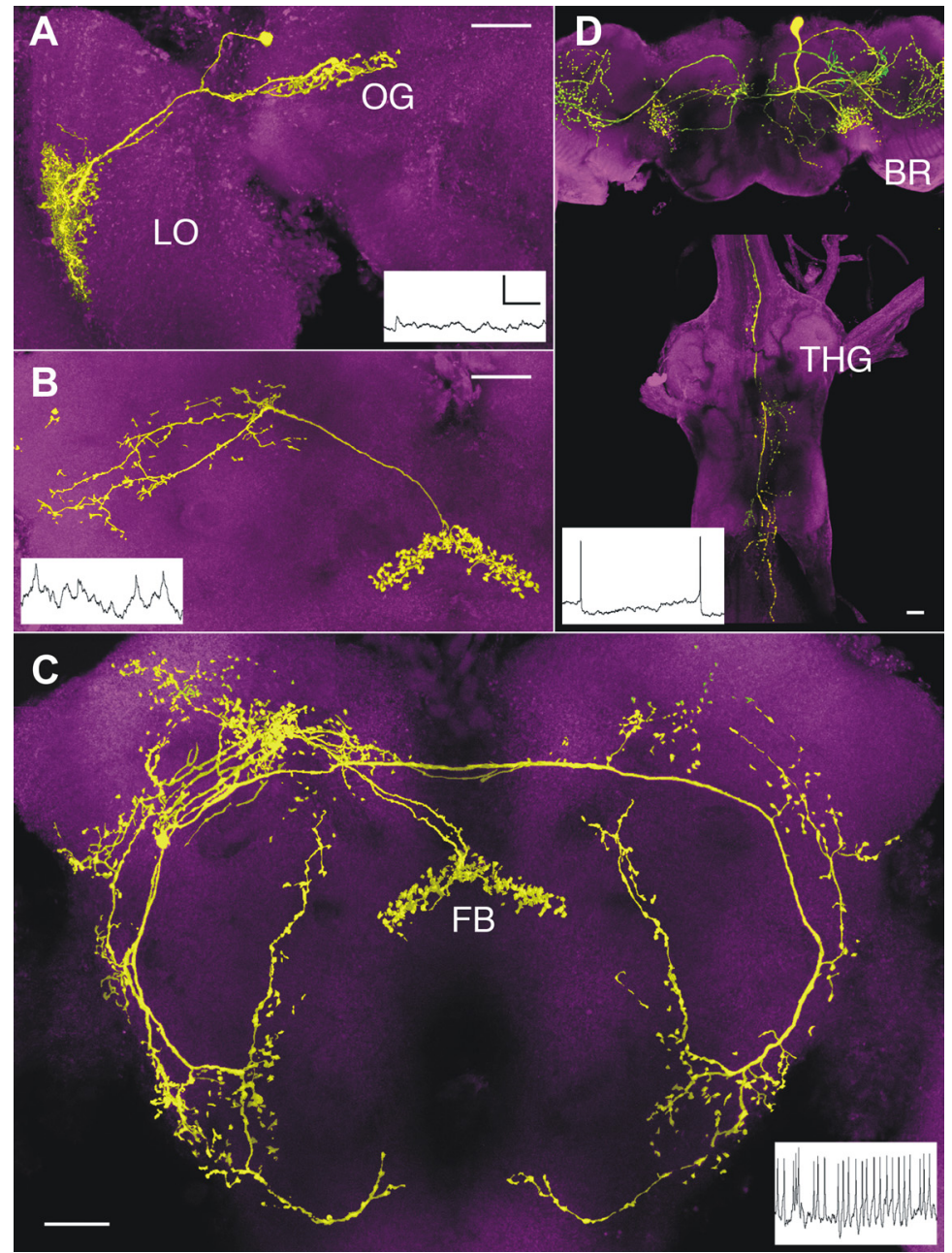

Figure 4. Scanning confocal micrographs of nonspiking and spiking neurons show that anatomy correlates with information transfer (insets). $A, A$ nonspiking LCN with a terminal in a dorsoanterior optic glomerulus (OG). Axon lengths, $80-90 \mu \mathrm{m}$ diameters $<0.5 \mu \mathrm{m} . \boldsymbol{B}$, One of a bilateral pair of uniquely identifiable protocerebral interneurons associated with the fan-shaped body (FB) of the central complex, showing mixed membrane potential fluctuations and action potentials. Axon lengths, $70-80$ $\mu \mathrm{m}$; diameters, $\sim 0.5 \mu \mathrm{m}$. C, One of a pair of uniquely identifiable spiking interneurons associated with the $F B$ of the central complex and extensions to protocerebral and deutocerebral regions. Axon lengths, 395- $410 \mu \mathrm{m}$; diameters, 1.0 $-1.5 \mu \mathrm{m} . \boldsymbol{D}, \mathrm{A}$ spiking descending neuron linking the protocerebrum of the brain (BR) to thoracic ganglia (THG). Axon length, $500-700 \mu \mathrm{m}$; diameters, 1.5-3 $\mu \mathrm{m}$. Scale bars, $20 \mu \mathrm{m}$. Calibration: $\boldsymbol{A}-\boldsymbol{C}, 5 \mathrm{mV} / 500 \mathrm{~ms} ; \mathbf{D}, 10 \mathrm{mV} / 500 \mathrm{~ms}$. L0, Lobula.

glomerulus (Fig. 5D). A similar functional organization has been demonstrated from recordings of the larger fly, Phaenicia sericata, in which a single LPL input to an identified glomerulus was broadly tuned to the orientation of a moving bar, whereas LINs associated with that glomerulus showed narrow tuning, as did the projection neuron from this glomerulus (Strausfeld et al., 2007).

\section{Electrophysiological properties of a single L1CN}

We next asked, if LCNs singly provide unreliable signals, might groups of the same neuronal clone, converging into a glomerulus, provide a more reliable signal, as suggested in the previous section? To test this, recordings were made from L1CNs, which have even smaller axon diameters than the LPL2CN described above but whose cell bodies are located closer to the surface of the brain and, therefore, are more accessible for whole-cell patch-clamp recording. Each $\mathrm{L} 1 \mathrm{CN}$ of the clone of $\sim 40$ neurons has an axon diameter $<0.5 \mu \mathrm{m}$ (Fig. $6 A$ ). Such neurons show both fast and slow spontaneous membrane potential fluctuations, seemingly independently of visual stimuli (Fig. $6 B$ ), as might be expected in neurons that receive many synaptic inputs conveying a range of visual information. Indeed, L1CN dendritic ensembles in the lobula are postsynaptic to the terminals of hundreds of retinotopic relay neurons from the medulla. To test whether the origin of spontaneous signals is from active presynaptic inputs, TTX $(1 \mu \mathrm{M})$ was applied to the perfusion saline. Membrane potential fluctuations decreased substantially, and the averaged power of fluctuations significantly decreased (Student's $t$ test, $n=20$; $p<0.05$ ) (Fig. 6C). To confirm further that the nonspiking feature found in the LCN was not caused by limitations of our recording technique, we compared voltageclamp and current-clamp recordings from Kenyon cells of the mushroom bodies and L1CNs (Fig. 6D,E). Kenyon cells of Drosophila are spiking neurons with 2- to $3-\mu \mathrm{m}$-diameter somata (Turner et al., 2008). Action potentials were initiated in the Kenyon cells when injecting positive current (Fig. 6Di), and inward active currents were visible in voltage-clamp recordings (Fig. 6Dii). In contrast, no action potentials or inward active currents were found in L1CNs (Fig. 6Ei,Eii), under identical physiological conditions, confirming the nonspiking nature of L1CNs.

\section{Responses of L1CNs to slow flicker stimuli}

The electrophysiological responses of L1CNs, like those of LPL2CNs, are best resolved by examining membrane potential fluctuations using power spectrum analyses. L1CNs comprise an assembly of 36-40 individual L1CNs, the axons of which project to the same optic glomerulus. The responses of an individual L1CN to slow flicker $(0.5 \mathrm{~Hz})$ are ambiguous because they are embedded within a background of synaptic activity, as shown by the four successive recordings in Figure 7Ai. An averaged time-frequency plot of these four recordings (Fig. 7Aii) and their calculated averaged power $(2-80 \mathrm{~Hz})$ (Fig. 7Aiii) show that, for a single L1CN, low-frequency oscillations mark the response of the neuron to light ON and light OFF.

However, if sibling neurons encode the same visual primitives, the summed responses from many L1CNs might be expected to show clearer evidence of responses to a defined visual stimulus. This is confirmed by averaging the responses from 33 identical L1CNs (Fig. 7B). The averaged responses to the first cycle of stimulation show a clear power increase to light $\mathrm{ON}$ (from Fig. 7Bii, a, b), followed by a decrease $\sim 200 \mathrm{~ms}$ thereafter (Fig. 7Bii, c). Similarly, a light OFF stimulus initiates a longer duration power increase for $\sim 700 \mathrm{~ms}$ (Fig. $7 \mathrm{Bii}, \mathrm{c}, \mathrm{d}$ ), followed by a decrease (Fig. 7 Bii, e). Such power changes during the first cycles of slow flicker are significant $(N=33 ; p<0.05)$, but subsequent flicker stimulation elicited no further responses, suggesting that L1CNs quickly adapt to the full-field flicker stimuli. 

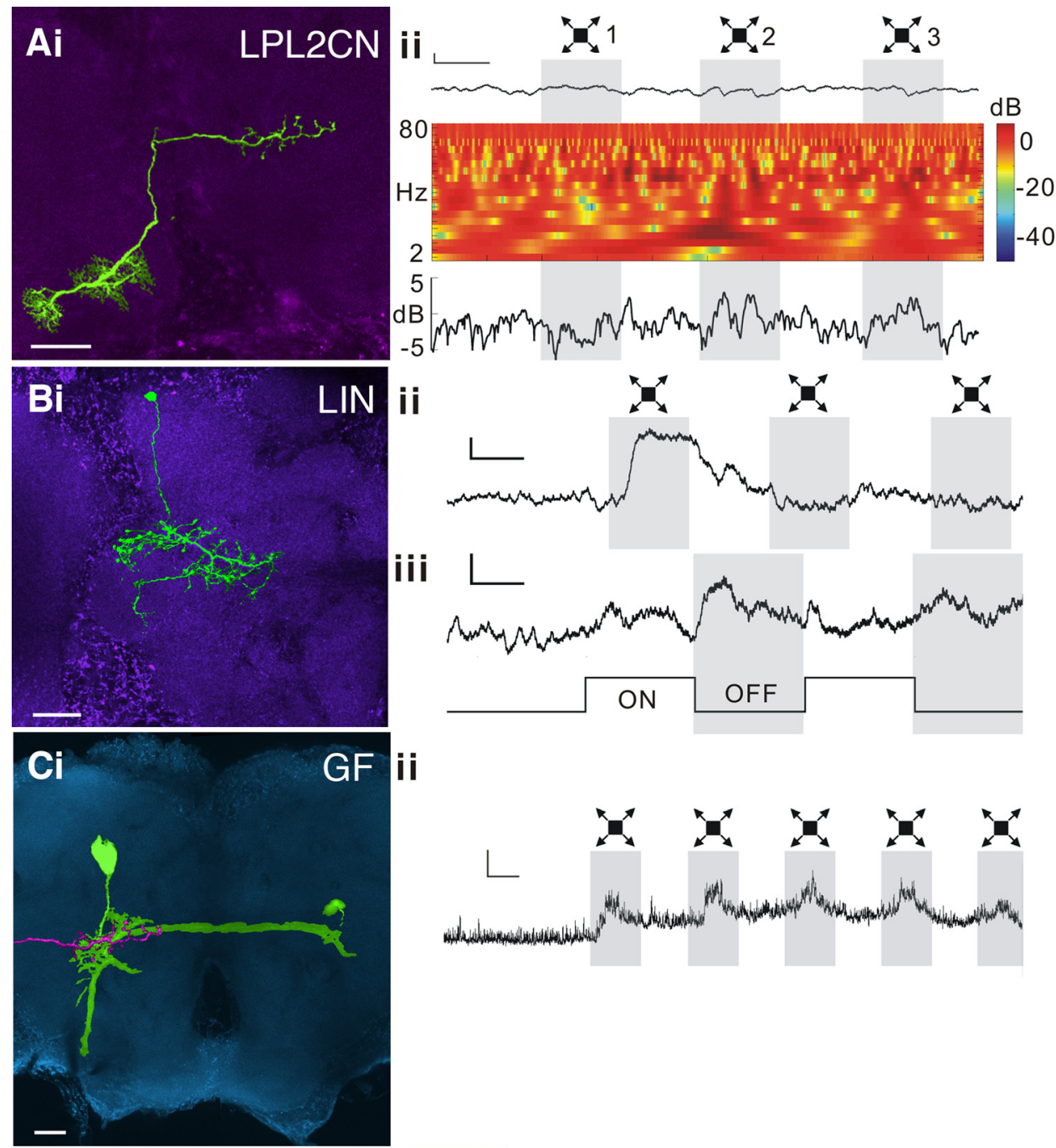

ii
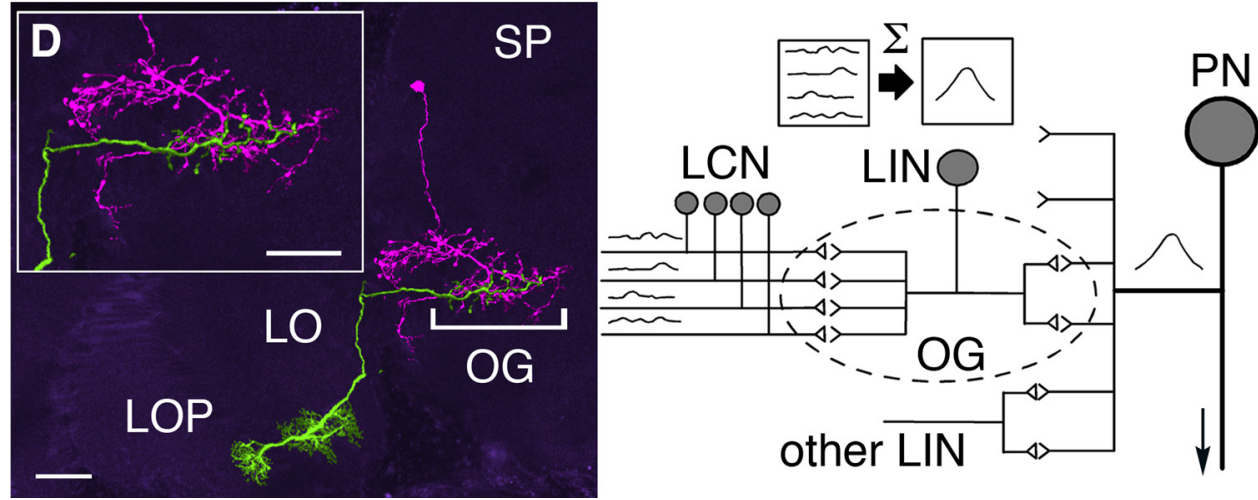

Figure 5. Neural integration enhances sensitivity to looming stimuli. A-C, Left, Confocal images of recorded neurons. Scale bars, $20 \mu \mathrm{m}$. Right, Corresponding recordings. Calibration: $2 \mathrm{mV} / 500 \mathrm{~ms}$. Aii, Power spectrum analysis illustrates thenonspiking LPL2CN responding to thelooming stimuli2 and 3. The first trace is the recording sample. The time-frequency plotin the middle shows the power of membrane potential oscillations calculated from the recording sample above. The line plot at the bottom shows averaged powers $(2-80 \mathrm{~Hz}$ ) throughout the stimulus calculated from the time-frequency plot above. $\boldsymbol{B}$, The unambiguous and rapidly adapting responses to looming and full-field flicker stimuli of the LIN in the GF glomerulus. C, The GF and its depolarizing responseto looming stimuli. An image of the terminal of $L P L 2 C N$ (pink) is superimposed on the GF dendrites to indicate their overlap in the GF glomerulus. D, Convergent processing in the optic glomerulus. Left, Montage showing overlap at the same optic glomerulus [bracketed optic glomerulus (OG)] of the recorded LIN (pink) and the axon terminal of a recorded LPL (green). This species of neuron, LPL2CN, belongs to the class of LPLs characterized by their dendrites in the lobula (LO) and lobula plate (LOP). Inset, Enlargement of the related glomerulus. Right, Schematic to illustrate convergence of LCNs to an OG. Responses of the LCNs are summed $(\Sigma)$ and carried by the LIN relaying to its cognate projection neuron (PN). PNs of $O G$ receive additional LIN inputs.

Direction and orientation selectivity of L1CNs to moving and static patterns

Studies of the dipteran $P$. sericata showed that specific types of LCNs selectively encode bar orientation and motion (Okamura and Strausfeld, 2007). We tested responses of individual L1CNs and subsets of L1CNs to moving bar stimuli, square-wave gratings, and sinusoidal gratings. L1CNs displayed directional selectivity to the oriented motion of a single bar (Fig. 8). Power 

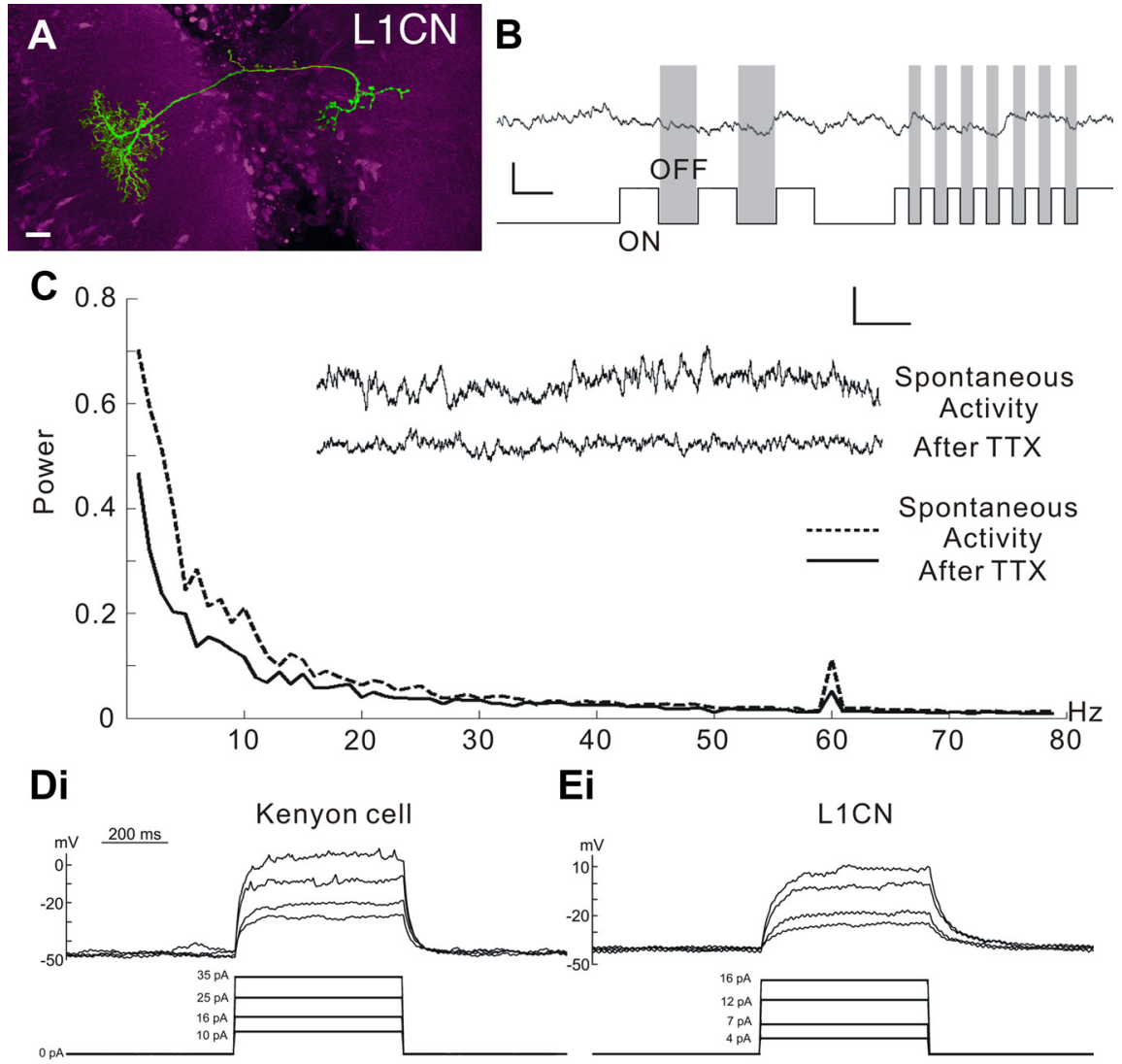

$\mathrm{Ei}$
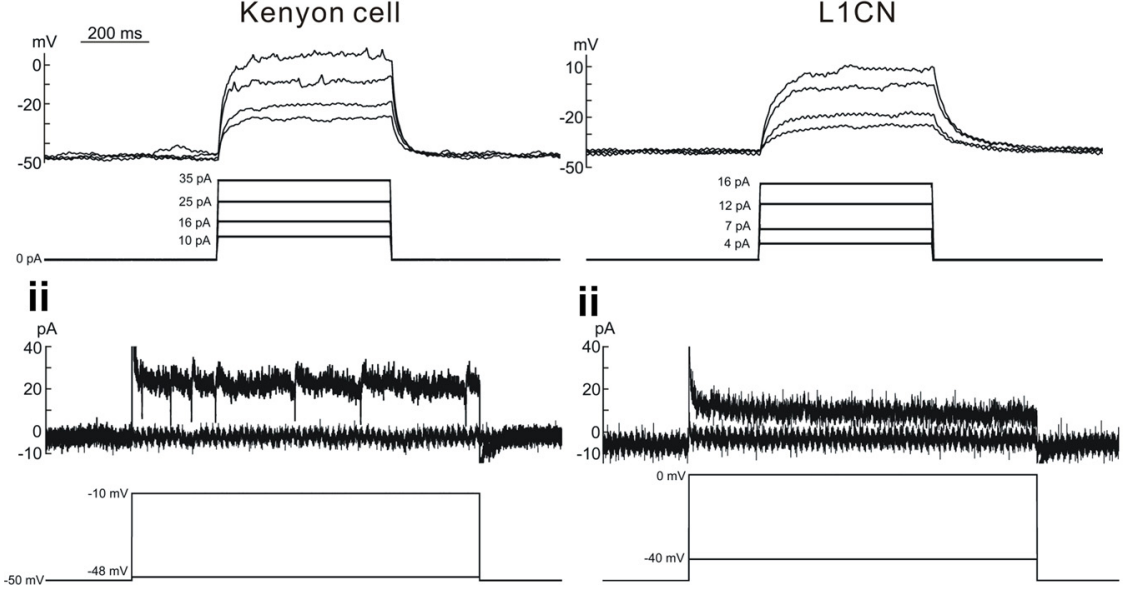

Figure 6. The nonspiking nature of a single L1CN labeled in the GAL4 line NP3045. A, A single recorded and biocytin-filled LCN. Scale bar, $10 \mu \mathrm{m} . \boldsymbol{B}$, Responses of a single L1CN showing typical nonspiking fast and slow membrane potential fluctuations, which appear to be unrelated to the visual ON/OFF stimuli. C, Plot showing averaged power fluctuations before and after applying TTX to the lobula. Inset, Sample recordings before and after application. Applying TTX reduces both fast and slow membrane potential fluctuations. D, E, Current-clamp (Di, Ei) and voltage-clamp (Dii, Eii) recordings from a Kenyon cell (D) and an L1CN cell (E). Action potentials and inward voltage active currents can be initiated in Kenyon cells but not in L1CN cells.

spectrum analysis shows that a single $\mathrm{L} 1 \mathrm{CN}$ has a subtle response to downward motion $\left(270^{\circ}\right)$ of a single bar (Fig. $8 \mathrm{Ai}$ ). The averaged power spectrum of $25 \mathrm{~L} 1 \mathrm{CN}$ s to the same stimulus sequence $200 \mathrm{~ms}$ before and $200 \mathrm{~ms}$ after the onset of bar motion for four different directions reveals that downward $\left(270^{\circ}\right)$ motion indeed initiates a significant response (Fig. $8 B, C ; N=25 ; p<0.05$ ). This shows that although a summed output signal from several L1CNs is robust, responses of a single $\mathrm{L} 1 \mathrm{CN}$ to visual stimuli are subtle and variable. Additionally, the polar plot (Fig. $8 D$ ) suggests that the preferred direction for L1CNs is $\sim 315^{\circ}$. The power spectrum analysis does not differentiate the EPSPs and IPSPs. Therefore, this result indicates either increased EPSPs or increased IPSPs in L1CNs at the preferred motion direction and, thus, the increased activities of upstream medulla inputs. Unlike the wide-field tangential cells in the lobula plate, which detect the direction of either horizontal or vertical motion, L1CNs showed no evidence of a significant "null" direction, which suggests the upstream medulla inputs were not significantly inhibited at any motion direction.
In contrast to the directional selectivity to the movement of a single bar stimulus, neither single L1CNs nor averaged subsets of $28 \mathrm{~L} 1 \mathrm{CNs}$ showed significant responses ( $p>0.05)$ to a square-wave grating moving in any of eight presented directions (Fig. 9A). Similarly, a sinusoidal grating moving in any of four different presented directions failed to elicit any specific response $(N=26 ; p>0.05)$ (Fig. $9 B$ ). Finally, we examined the orientation selectivity of L1CNs to static square-wave gratings oriented at four different angles, at increments of $45^{\circ}$. Recordings from individual L1CNs showed no clear response to any static pattern. Furthermore, the power spectrum of the averaged response of $28 \mathrm{~L} 1 \mathrm{CNs} 200 \mathrm{~ms}$ before and $200 \mathrm{~ms}$ during stimulus presentation also did not show significant response for static gratings at any specific orientation $(N=28$; $p>0.05$ ) (Fig. 9C).

\section{Discussion}

Axon diameters and signal reliability Retinotopic output neurons from the lobula of Drosophila, which have axon diameters of $\leq 0.5 \mu \mathrm{m}$, do not transmit action potentials. This is typical of the many small interneurons in the insect visual system that are arranged as repeat ensembles. However, mushroom body intrinsic neurons (Kenyon cells) may be an exception to this because few of them, at any time, are required to accurately encode odorant identity through the mechanism of "sparsening" (Wang et al., 2004).

In the dipteran Phormia, relays connecting the medulla to the lobula and lobula plate have axon diameters of between 0.5 and $3 \mu \mathrm{m}$. These neurons generally respond with graded potentials (Douglass and Strausfeld, 1995, 1996, 2003) as do the larger axon diameter (2-4 $\mu \mathrm{m})$ lamina monopolar cells, which extend from the lamina to the medulla (Autrum et al., 1970; Zettler and Järvilehto, 1973). Even the 15- $\mu \mathrm{m}$-diameter axons of "giant" motion-sensitive neurons in the lobula plate can conduct by graded potentials in addition to spiking responses. However, there are some exceptions. In the hoverfly Eristalis tenax, objectdetecting neurons relaying from the lobula show clear spiking responses, as do neurons in Phormia that respond to moving bars (Nordström et al., 2006; Okamura and Strausfeld, 2007).

Signal reliability is also critical for neurons that occur as single pairs of uniquely identifiable neurons or as very small populations in the brain, or small subsets of Kenyon cells, each subset encoding an odorant. In Drosophila, such neurons conduct by spikes or by mixed codes: membrane potential fluctuations and action potentials. Examples are the widefield, directional, selective tangential cells of the lobula plate, which occur either as a uniquely identifiable set of three horizontal motion-sensitive neurons or as 11 uniquely distinct VS neurons that collaborate to mediate responses to changes in optic flow (Borst and Haag, 

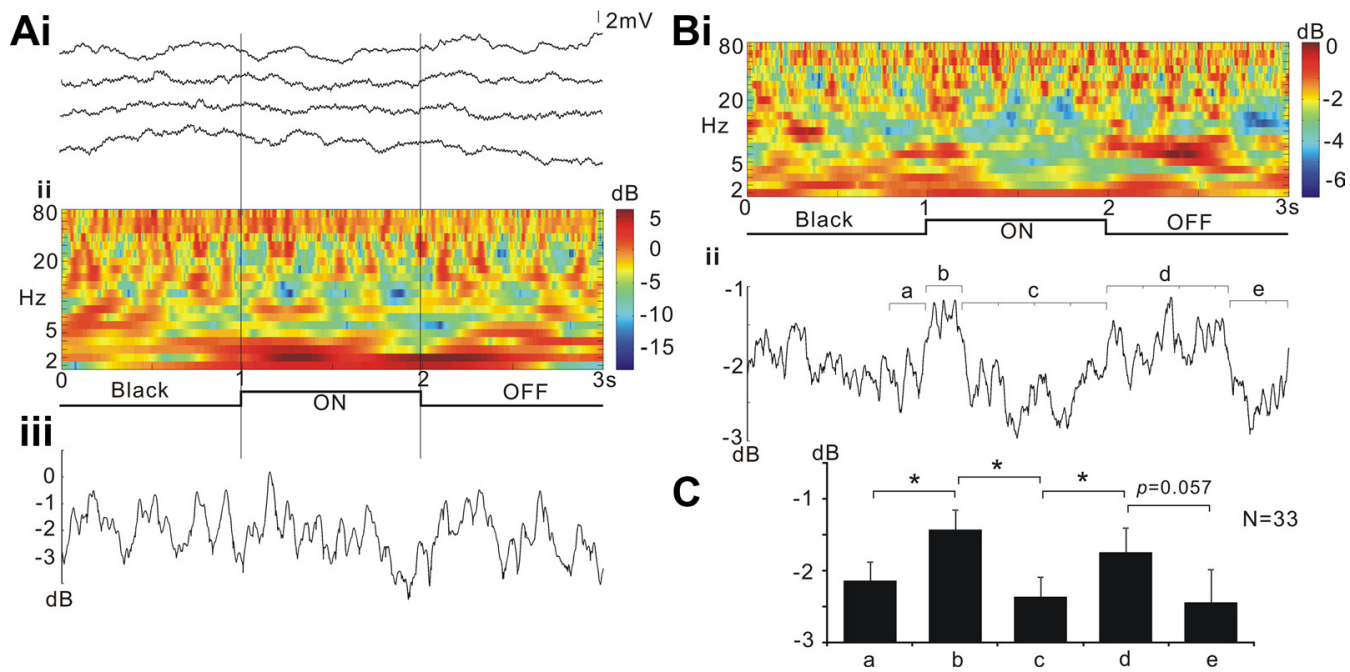

Figure 7. L1CNs respond to slow flicker. A, Responses of a single L1CN. Ai, Membrane potential recordings in four successive trials. Aii, Time-frequency plot showing the power of membrane potential oscillations during the stimulus, averaged from the four trials shown in Ai. Aiii, Line plot showing averaged powers $(2-80 \mathrm{~Hz})$ throughout the stimulus calculated from the time-frequency plot of Aii. This L1CN showed weak response to slow flicker. $B$, Time-frequency plot of averaged response from grouped L1CNs $(N=33)$. Letters indicate time windows where the averaged powers were statistically compared (Bii: a, b, $200 \mathrm{~ms} ;$ c, $800 \mathrm{~ms} ; \mathrm{d}, 700 \mathrm{~ms} ; \mathrm{e}, 300 \mathrm{~ms}$ ). C, Averaged decibel power at various time windows during the stimulus (mean \pm SEM). Both light $0 \mathrm{~N}$ and light $0 \mathrm{FF}$ initiate significantly increased power of membrane potential fluctuations. ${ }^{*} p \leq 0.05$.
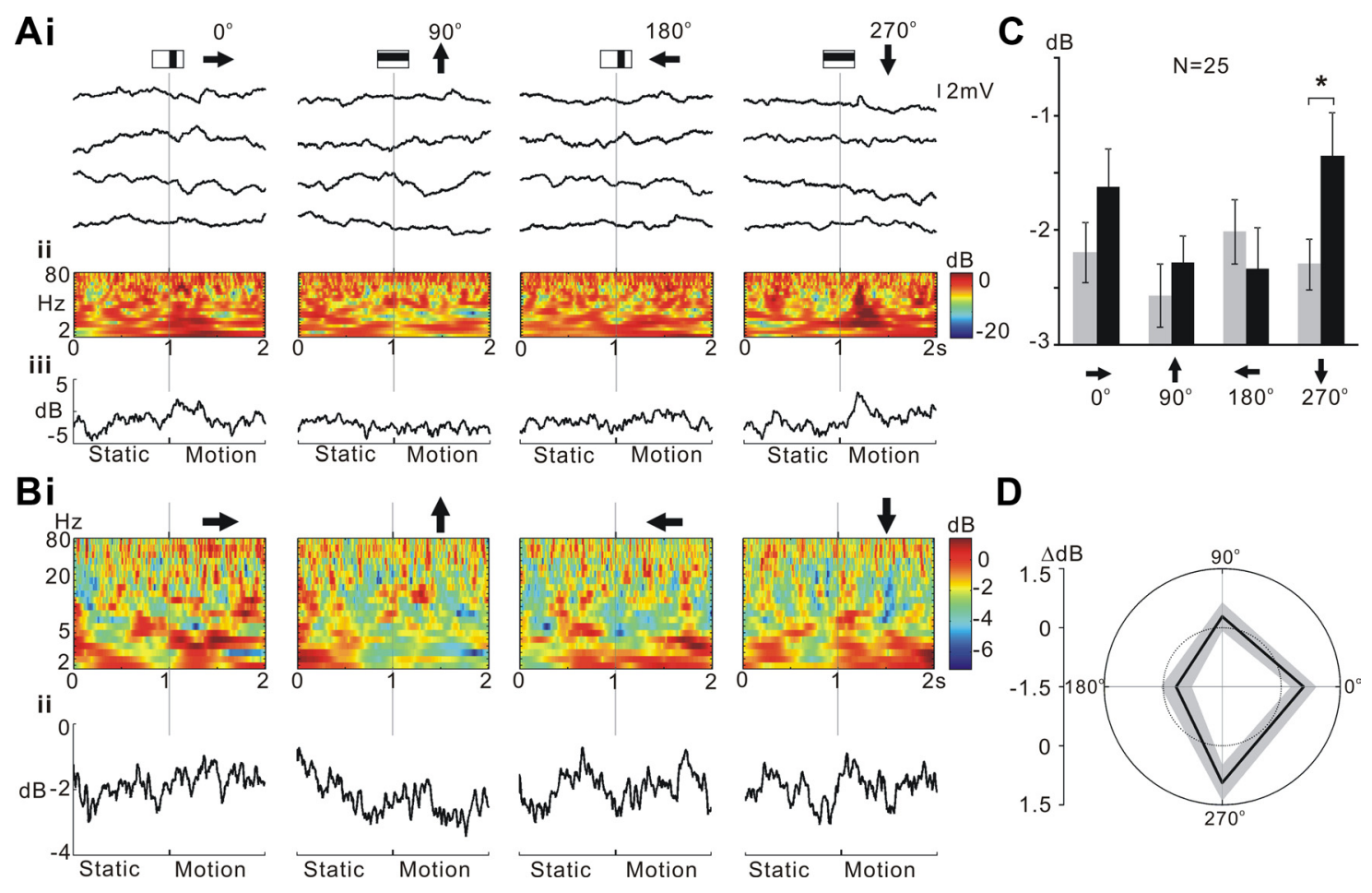

Figure 8. L1CNs respond selectively to a single bar moving in a downward direction. $A$, Responses to single-bar motion of an individual L1CN. Ai, Sample recordings in four successive trials for each stimulus direction. Aii, Time-frequency plots during the stimulus averaged from each of the four sets of trials shown in Ai. Aiii, Line plots showing the mean power (2-80 $\mathrm{Hz})$ change throughout the stimulus for each stimulus direction. $\boldsymbol{B}$, Time-frequency plot of averaged responses from grouped LCNs $(N=25)$ for each direction. $B$ ii, Line plots show the averaged power $(2-80 \mathrm{~Hz})$ change throughout the stimulus calculated from the time-frequency plots in Bi. Arrows, Direction of the motion pattern with respect to the head of the fly. $C$, Mean power during $200 \mathrm{~ms}$ before (gray bar) and after (black bar) motion stimulus onset for different directions (mean \pm SEM). Downward $\left(270^{\circ}\right)$ motion initiated a significant response $(p \leq 0.05)$. D, Polar plots of mean power difference between $200 \mathrm{~ms}$ before and after motion stimulus onset for different directions. Gray area, Mean \pm SEM; inner dotted line, zero power change.

1996). Neurons with long axons, such as the unique pairs of interneurons linking the central body with many areas of the lateral protocerebrum and deutocerebrum or those that carry data from the brain to thoracic ganglia, also invariably conduct by spikes.

In contrast to uniquely identifiable pairs or small clones of neurons belonging to the midbrain, many neurons in the optic lobes occur as ensembles of identical, clonally related neurons. In the medulla of Drosophila and other fly species, there are $\sim 50$ types of retinotopic neurons, spaced one to each retinotopic column (Fischbach and Dittrich, 1989; Bausenwein et al., 1992). In the lobula, there are $\sim 15$ different clones of output neurons, each of which comprises an ensemble of $\sim 40$ identical neurons (Otsuna and Ito, 2006). Each neuron of an ensemble subtends 
$\mathbf{A}_{\mathrm{dB}}$

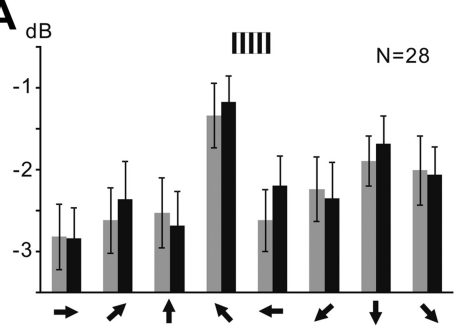

B

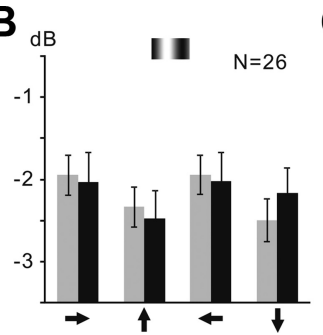

Figure 9. LICNs do not show significant responses to directional square-wave gratings motion, sinusoidal gratings motion, and presenting static square-wave gratings. The bar graphs show the mean power in $200 \mathrm{~ms}$ before (gray bar) and $200 \mathrm{~ms}$ after (black bar) the beginning of the motion stimuli at different directions or displaying static patterns at the different orientations (mean \pm SEM). Arrows, Direction of the motion pattern with respect to the head of the fly. $A$, Square-wave gratings moving in eight different directions $(N=28 ; p>0.05)$. B, Sinusoidal gratings moving in four different directions $(N=26 ; p>0.05)$. C, Static square-wave gratings at four different orientations $(N=28 ; p>0.05)$.

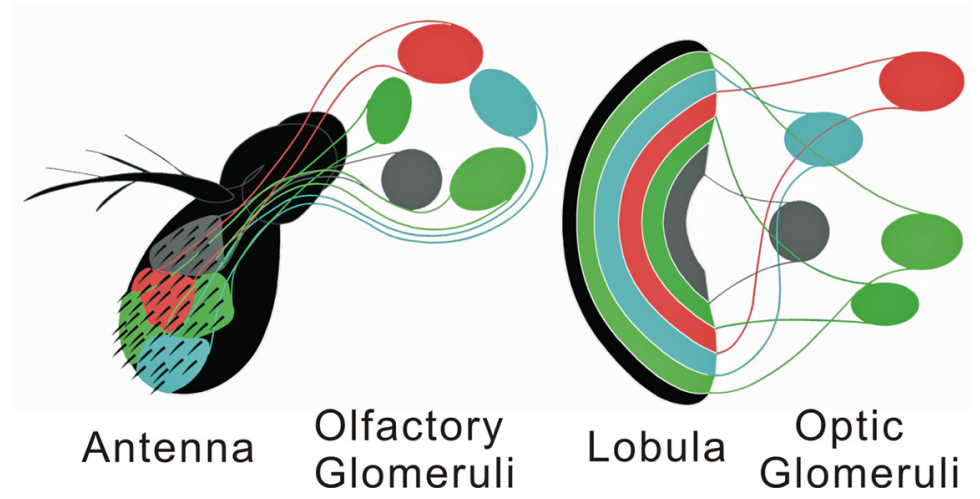

Figure 10. Schematic comparing central segregation of coded channels to olfactory and optic glomeruli. Olfactory receptor neurons encoding data about specific ligands segregate to unique olfactory glomeruli, 40 of which are located in the Drosophila deutocerebrum (Laissue et al., 1999). Each genetically defined clone of lobula outputs with dendrites in specific layers of the lobula encodes data about specific visual primitives. Axons from each clone segregate to unique optic glomeruli, 18 of which are found in the Drosophila protocerebrum (Otsuna and Ito, 2006).

6-9 visual sampling units of the retina and has dendrites, and thus receptive fields (Okamura and Strausfeld, 2007), that overlap with a surround of at least 8-12 neurons of the same clonal identity (Strausfeld and Hausen, 1977; Strausfeld and Gilbert, 1992). This anatomical arrangement ensures that $8-12$ neurons of the same clone view the same part of the visual field.

In Drosophila, such outputs from the lobula have extremely thin axons, and these cells conduct by graded potentials. Each LCN exhibits significant membrane voltage fluctuations, which likely reflect the many postsynaptic sites from medulla afferents. An important finding of this study was that recordings from many single L1CNs show that none reliably encodes a visual primitive, whereas the summed responses of L1CNs show clear responses to defined visual stimuli. Thus, since any ensemble of LCNs converges at its unique glomerulus, it is expected that a subset of LCNs will respond to a given visual stimulus and that the summed responses of this subset would drive postsynaptic neurons of their target glomerulus. In larger dipterans, it has also been shown that different optic glomeruli respond to different visual primitives (Okamura and Strausfeld, 2007; Strausfeld et al., 2007).

It is conceivable that the weak responses we recorded in individual LCNs is attributed to the long electrotonic distance between soma and axon. However, there are two major reasons to reject the idea that these nonspiking characteristics are artifactual. First, using an identical recording methodology, small spiking neurons in the midbrain were also shown to have long, thin

$\mathbf{C}_{\mathrm{dB}}$

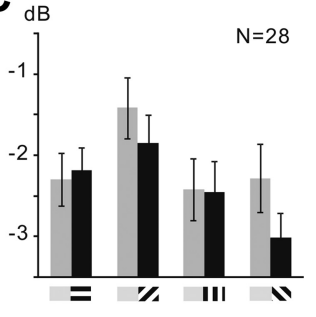

neurites between their cell bodies and their main integrative regions. Second, recordings of the smallest retinotopic neurons in the medulla of a larger fly species, Phaenicia sericata, consistently showed that they encode data in a nonspiking fashion, regardless of the location of the electrode in the neuron (Douglass and Strausfeld, 2003).

\section{Integration at the optic glomerulus}

If an individual output neuron from the lobula complex can have subtle and variable responses to specific visual stimuli but the summed responses of a subset of LCNs belonging to the same clone show a clearer response, might local interneurons postsynaptic to their terminals in their relevant optic glomerulus integrate input signals and unambiguously respond to the same visual stimuli? Recordings of a LIN in the GF optic glomerulus complex suggest this is case: the LIN responds unambiguously to a looming stimulus, whereas the response of the single LPL2CN to the same stimulus can be resolved only from a power spectrum analysis. However, as shown above, when responses of many of the same type of lobula output neurons are summed, their collective response is unambiguous.

The GF glomerulus receives LPL2CN inputs and contains LIN processes as well as one major dendritic process of the GF (Bacon and Strausfeld, 1986). The GF glomerulus LIN responds to looming stimuli and responds to intensity decrements. Looming stimuli activate the LPL2CN inputs of the GF glomerulus. Responses by the LIN are also the same as those that drive the GF. That the LIN rapidly adapts to looming stimuli whereas GF does not suggests that several LINs are associated with the glomerulus and that these may recruit signals from successive groups of activated LPL2CN afferents. Though it remains to be demonstrated that the LPL2CN clone is presynaptic to the LIN and the GF, there is strong evidence in larger dipteran species that the Col A afferents, which also converge on the GF glomerulus, are directly presynaptic to the GF. For example, electron microscopy studies have shown that in Musca domestica Col A cells establish electrical synapses onto the GF (Strausfeld and Bassemir, 1983), and cobalt is introduced into the GF passes, rather spectacularly, into the entire array of Col A afferents (Strausfeld and Bacon, 1983; Bacon and Strausfeld, 1986). Col A cells in P. sericata respond with graded potentials to decrements in illumination and to movement of edges (Gilbert and Strausfeld, 1992). The convergence of $\mathrm{Col}$ A neurons and neurons of the LPL2CN clone at the GF glomerulus does suggest that there is a more complex control system eliciting GF responses than has been hitherto envisaged.

\section{Evolutionary considerations: segmental correspondence with the olfactory system}

The convergence of axons from a clone of optic lobe outputs to an optic glomerulus suggests a mechanism that establishes reliable downstream responses: one or more local interneurons of the 
glomerulus complex integrate, and average inputs from members of an isomorphic population of retinotopic relay neurons from the lobula complex (Fig. 5D). Recordings from the GF glomerulus show that its LIN responds reliably to the same looming stimulus that drives the LPL2CN afferent supply to that glomerulus. The demonstration that the LIN response is relatively noise free suggests that one function of LINs is to disambiguate information carried by afferents to a glomerulus from synaptic noise generated at the dendritic trees within the lobula. Noise-free information could then be relayed by the LIN to the projection neurons of the glomerulus. These are of two types: premotor descending neurons such as the GF, which project to the thoracic ganglion; and relay neurons, which project to higher centers in the brain such as the dorsal protocerebral lobes (Strausfeld and Okamura, 2007) and their connections to the central complex (Liu et al., 2006).

This convergence of lobula outputs to uniquely identifiable optic glomeruli in the brain's first segment, the protocerebrum, is comparable to the convergence of olfactory sensory neurons (OSNs) to antennal lobe glomeruli in the brain's second segment, the deutocerebrum (Fig. 10), where each unique glomerulus in the fly's antennal lobe is targeted by the axons of a specific set of OSNs on the antenna, which expresses a particular olfactory receptor protein (Vosshall et al., 2000). In the antennal lobe, noisy signals from OSNs are refined by local interneurons and then relayed to higher centers by projection neurons (Laurent, 2002; Wilson, 2008). The present results provide electrophysiological evidence that noisy signals in an isomorphic population of lobula outputs are similarly refined by local interneurons of the optic glomerular complex. Therefore, reliable responses in an optic glomerulus are established through convergence and signalaveraging processes.

The present results further support the proposition that the optic glomerular complex and the antennal lobes are serially homologous neural systems having the same principal anatomical and functional organization and with the common function of refining and integrating incoming signals (Strausfeld et al., 2007). Glomerular organization in the protocerebrum and deutocerebrum reflects a ground pattern that can be identified in every ganglion of the CNS (Strausfeld, 2012). Throughout, each type of receptor, representing one or another modality, sends its axon to a specific domain in the relevant ganglion. These domains-in some ganglia represented by glomerular volumes, in others by allantoid or ovoid volumes-are connected by spiking and nonspiking local interneurons that integrate the sensory input and relay behaviorally meaningful information to central neuropils and to motor circuits (Burrows, 1996). Such arrangements evolutionarily derive from an ancestral ground pattern seen in archaic arthropods, each segment of which was composed of identical elements (Strausfeld, 2012). As demonstrated by the protocerebrum and deutocerebrum, present day insects reflect this ancestral ground pattern even in the brain, despite each segment having evolved its unique sensory configuration.

\section{References}

Autrum H, Zettler F, Järvilehto M (1970) Postsynaptic potentials from a single monopolar neuron of the ganglion opticum I of the blowfly Calliphora. J Comp Physiol A 70:414-424.

Bacon JP, Strausfeld NJ (1986) The dipteran 'Giant fibre' pathway: neurons and signals. J Comp Physiol A 158:529-548.

Bausenwein B, Dittrich AP, Fischbach KF (1992) The optic lobe of Drosophila melanogaster. II. Sorting of retinotopic pathways in the medulla. Cell Tissue Res 267:17-28.

Borst A, Haag J (1996) The intrinsic electrophysiological characteristics of fly lobula plate tangential cells. I. Passive membrane properties. J Comput Neurosci 3:313-336.

Burrows M (1996) The neurobiology of an insect brain. Oxford: Oxford UP. Cohen MX, David N, Vogeley K, Elger CE (2009) Gamma-band activity in the human superior temporal sulcus during mentalizing from nonverbal social cues. Psychophysiology 46:43-51.

Douglass JK, Strausfeld NJ (1995) Visual motion detection circuits in flies: peripheral motion computation by identified small-filed retinotopic neurons. J Neurosci 15:5596-5611.

Douglass JK, Strausfeld NJ (1996) Visual motion-detection circuits in flies: parallel direction- and non-direction-sensitive pathways between the medulla and lobula plate. J Neurosci 16:4551-4562.

Douglass JK, Strausfeld NJ (2003) Retinotopic pathways providing motionselective information to the lobula from peripheral elementary motiondetecting circuits. J Comp Neurol 457:326-344.

Faisal AA, Laughlin SB (2007) Stochastic simulations on the reliability of action potential propagation in thin axons. PLoS Comput Biol 3:e79-91.

Fischbach KF, Dittrich APM (1989) The optic lobe of Drosophila melanogaster. I. A Golgi analysis of wild-type structure. Cell Tissue Res 258:441-475.

Gao Q, Yuan B, Chess A (2000) Convergent projections of the Drosophila olfactory neurons to specific glomeruli in the antennal lobe. Nat Neurosci 3:780-785.

Gilbert C, Strausfeld NJ (1992) Small-field neurons associated with oculomotor and optomotor control in muscoid flies: functional organization. J Comp Neurol 316:72-86.

Gu H, O’Dowd D (2007) Whole cell recordings from brain of adult Drosophila. J Vis $\operatorname{Exp}(6): 248$.

Heisenberg M, Wolf R (1984) Vision in Drosophila: genetics of microbehavior, pp 17, New York: Springer.

Holm S (1979) A simple sequentially rejective multiple test procedure. Scand J Statist 6:65-70.

Joesch M, Plett J, Borst A, Reiff DF (2008) Response properties of motionsensitive visual interneurons in the lobula plate of Drosophila melanogaster. Curr Biol 18:368-374.

Koto M, Tanouye MA, Ferrus A, Thomas JB, Wyman RJ (1981) The morphology of the cervical giant fiber neuron of Drosophila. Brain Res 221:213-217.

Laissue PP, Reiter C, Hiesinger PR, Halter S, Fischbach KF, Stocker RF (1999) Three-dimensional reconstruction of the antennal lobe in Drosophila melanogaster. J Comp Neurol 405:543-552.

Laurent G (2002) Olfactory network dynamics and the coding of multidimensional signals. Nat Rev Neurosci 3:884-895

Liu G, Seiler H, Wen A, Zars T, Ito K, Wolf R, Heisenberg M, Liu L (2006) Distinct memory traces for two visual features in the Drosophila brain. Nature 439:551-556.

Maimon G, Straw AD, Dickinson MH (2010) Active flight increases the gain of visual motion processing in Drosophila. Nat Neurosci 13:393-399.

Marr D (1976) Early processing of visual information. Philos Trans R Soc Lond B Biol Sci 275:483-519.

Nordström K, Barnett PD, O'Carroll DC (2006) Insect detection of small targets moving in visual clutter. PLoS Biol 4:e54.

Okamura JY, Strausfeld NJ (2007) Visual system of calliphorid flies: motion- and orientation-sensitive visual interneurons supplying dorsal optic glomeruli. J Comp Neurol 500:189-208.

Otsuna H, Ito K (2006) Systematic analysis of the visual projection neurons in Drosophila melanogaster. I. Lobula-specific pathways. J Comp Neurol 497:928-958.

Ramon y Cajal S, Sánchez SD (1915) Contribución al conocimiento de los centros nerviosos de los insectos, Pt 1, Retina y centros opticos, Vol 13, pp 1-168. Madrid: Trabajos del Laboratorio de Investigaciones Biológicas del Universidad.

Reiser MB, Dickinson MH (2008) A modular display system for insect behavioral neuroscience. J Neurosci Methods 167:127-139.

Sanes JR, Zipursky SL (2010) Design principles of insect and vertebrate visual systems. Neuron 66:15-36.

Schnell B, Joesch M, Forstner F, Raghu SV, Otsuna H, Ito K, Borst A, Reiff DF (2010) Processing of horizontal optic flow in three visual interneurons of the Drosophila brain. J Neurophysiol 103:1646-1657.

Strausfeld NJ (1970) Golgi studies on insect. II. The optic lobes of Diptera. Philos Trans R Soc Lond B Biol Sci 258:135-223. 
Strausfeld NJ (2012) Arthropod brains: evolution, functional elegance, and historical significance. Cambridge: Harvard UP.

Strausfeld NJ, Bacon J (1983) Multimodal convergence in the central nervous system of dipterous insects. In: Fortschritteder zoologie: multimodal convergence in sensory systems, Chap 28 (Horn E, ed), pp 47-76. New York: Gustav Fischer Verlag.

Strausfeld NJ, Bassemir UK (1983) Cobalt-coupled neurons of a giant fibre system in Diptera. J Neurocytol 12:971-991.

Strausfeld NJ, Gilbert C (1992) Small-field neurons associated with oculomotor control in muscoid flies: cellular organization in the lobula plate. J Comp Neurol 316:56-71.

Strausfeld NJ, Hausen K (1977) The resolution of neural assemblies after cobalt injection into neuropil. Proc R Soc Lond B 199:463-476.

Strausfeld NJ, Lee JK (1991) Neuronal basis for parallel visual processing in the fly. Vis Neurosci 7:13-33.

Strausfeld NJ, Okamura JY (2007) Visual system of calliphorid flies: organization of optic glomeruli and their lobula complex efferents. J Comp Neurol 500:166-188.
Strausfeld NJ, Sinakevitch I, Okamura JY (2007) Organization of local interneurons in optic glomeruli of the dipterous visual system and comparisons with the antennal lobes. Dev Neurobiol 67:1267-1288.

Turner GC, Bazhenov M, Laurent G (2008) Olfactory representations by Drosophila mushroom body neurons. J Neurophysiol 99:734-746.

Vosshall LB, Wong AM, Axel R (2000) An olfactory sensory map in the fly brain. Cell 102:147-159.

Wang Y, Guo HF, Pologruto TA, Hannan F, Hakker I, Svoboda K, Zhong Y. (2004) Stereotyped odor-evoked activity in the mushroom body of Drosophila revealed by green fluorescent protein-based $\mathrm{Ca}^{2+}$ imaging. J Neurosci 24:6507-6514.

Wilson RI (2008) Neural and behavioral mechanisms of olfactory perception. Curr Opin Neurobiol 18:408-412.

Wilson RI, Laurent G (2005) Role of GABAergic inhibition in shaping odorevoked spatiotemporal patterns in the Drosophila antennal lobe. J Neurosci 25:9069-9079.

Zettler F, Järvilehto M (1973) Active and passive axonal propagation of non-spike signals in the retina of Calliphora. J Comp Physiol A 85:89-104. 